\title{
HOW WOULD BE OUR GREAT - - - - GREAT GREAT SONS AND DAUGHTERS LOOKING IN REMOTE FUTURE?
}

\author{
A.K. SINGH ${ }^{1}$ \\ Genetics Laboratory, Department of Zoology, Institute of Science, Banaras Hindu University, Varanasi, U.P., India
}

So far the existence of life is concerned; at least our earth is the only planet of solar system where living beings are found in highly diversified form. Since our universe is very vast, it has millions of planetary clusters like our own; we can expect many more celestial platforms where life would definitely be flourishing. Since the time of origin of life on our planet, unimaginable types of variations occurred that resulted into differentiation of varied types of life, i.e., from single cell to multi-cellular types. Things like buried rocks, hard calcified biological materials to skeletal remains have been used as evidences to know the organic world that existed many millions years ago. In the long 3.5 billion years of organic history of evolution, we Homo sapiens are the finest product of this natural phenomenon. Our origin is anticipated about 5 million years ago from a common ancestor that gave rise to Chimpanzee and humans. In fact, during the entire journey of biological evolution, we have spent very little period on this planet, however, our extraordinary mental capability has helped us to understand the past and future happenings of major evolutionary events.

Those who understand evolutionary events do think curiously about their future generations, particularly, the major changes in their external appearance, intelligence, technological advances and physical ability. Evolutionary changes are ever going process. This influences every sort of life, whether microscopic organisms through giant mammals. We are, in fact, the consequence of evolutionary changes that has made us to prosper since the dawn of our origin. While advocating evolution of our own race, we talk about the existence of human species that existed from Ramapithecus to Australopithecus to Homo habilis to Homo erectus to Homo sapiens. Our children, while studying aspects of human evolution in their text books take this topic humorously when they see the pictures of different ancient human descendents. Such pictures are almost real as they have been built on the skeletal remains of our age old ancestors. The fossil remains obtained from the caves and from excavations are in fact subjected to radioactive dating which help us to know the age of such fossils and then, a picture appearance of the fossils give idea about the physical look of the individuals existing during those days. It is quite likely to think about our past and future. Regarding remote past, we try to accumulate scientific evidences to articulate a real story about our history. For future, may be for 500 or 1000 or 10,000 years or even more from now, only probable anticipation can be made which may be supported by some philosophical clues.

What distinct changes are likely to occur in the purview of physical appearance, physical and mental ability, family bonding and religious believes? Since evolution is an unstoppable process, the evolutionary changes in our own species are inevitable. Mutations in our gene pool would result into new traits. The evolutionary forces which result to change in gene frequency would cause a substantial change in the genetic makeup of our remote future populations. Some of the probable changes could be: Larger size of cranium accommodating more developed brain. Since the origin of humans, steady increase in the cranial capacity has been recorded. This trend is expected to occur due to directional selection. The future populations would be fairer due to loss of hairs. In humans, a number of morphological traits are rudimentary, which persisted in better developed structures in other mammals or animals from which we are expected to originate. The reduction in the size of such structures might have initiated in ancient time due to mutational changes in the genes determining their appearance. Such changes, in fact, did not influence the functioning and survival of its race and therefore allowed to happen. To answer that in future descendents, some more organs may undergo severe modification, cannot be ruled out. Based on this concept, one can expect substantial changes in the overall look of human face. Due to differences in the feeding materials and feeding habits, chin will recede and become shorter.

A remote future descendents would have most notable change in their skin colour. Due to day activity being confined to shelter places, the skin will get fairer. We are witnessing hectic activities going in the indoor places or people using skin protecting creams or cover throughout the 
world; it would leave its effect on the concentration and distribution of colour cells in our skin. Some believe that our future descendents would have higher occurrence of myopia (short sightedness) and allergies problems. Reasons for this have been suggested due to increasing optical activities requiring more stress and strain over iris muscles, cases of myopia will have higher frequency in the population and people will have allergic reactions more common due to their more hygienic living habits, consequently, they would not be exposed to varied kinds of allergens. Primates like gorilla, chimp or other apes have long pointed canines. Since the onset of humans, a steady decrease in the size of canines has been observed. Based on the feeding habits it is quite likely that our future races would have much degraded canines. Overall strength of our bite would be weaker due to our habituation to feed soft food materials. Some people even predict disappearance of little toes.

Whether in remote future, one can expect existence of two separate species of humans? To my consideration, it is a remote possibility or straight way, it can be said that no two species of humans will exist together on this planet. Due to globalization, mixing of different human races have enhanced in the present era than before and therefore speciation by splitting our existing species into two and being separated for a long period that no gene flow occur between them seems to be impossible. This may only happen if a separate population of us inhabit a new space (planet) and remain cutoff for a period that cause enough genetic variation leading to two distinct gene pools that refrain gene flow. It is scientifically documented that once upon a time, we lived with Neanderthal, but what really resulted the complete wiping out of Neanderthal, is one of the major puzzle that we answer by saying that intense fighting between Neanderthals and Homo sapiens sapiens resulted their elimination. Existing two wise communities on the same land simultaneously with almost similar needs would definitely cause intense conflict.

The growing intelligence of future human beings will make them evolve technologically, to which one can refer digitally. Their reliance on artificial intelligence will dominate than their relations. Everyone will have one's DNA profile which will assist physician to treat the person according to his or her individual characteristics. The field of nanotechnology would be assisting in the treatment of enumerable ailments including cancer. Transgenic organisms will be providing bizarre organic products for human utilization. Space would be one of the highly utilized spot from where a number of our activities at the ground will be monitored. Access to planetary bodies, particularly, mars will be most preferred endeavor for bringing raw materials from there to ground for our use. Advancement in the field of medical sciences would be unbelievable, especially with respect to organ transplant and stem cell therapy. Treatment of infectious diseases would be ever challenging due to evolution of highly resistant microorganisms, but unprecedented research, would help to overcome such challenges. Our own activity may harm us tremendously. Man- made disasters may result into global destructions resulting into nuclear winter and the survivors may have unique adaptability to nuclear radiations which can alter our lives drastically. There would be 100 percent literacy and people will have total faith in scientific advances that would lead to least affiliation for religious trust, superstitions and age old cultures. Very unexpected changes will be there with respect to family cohesion. This world has seen mass living of human community during ancient time period for mutual safety and immediate gain. Large combined families split into nuclear families in quite wide spread parts of this globe. Psychological set up of existing community indicates further dissection in the family forms leading to individuals living with profuse promiscuity in both sexes. Whether more distinct changes will appear between the two sexes of our species or sexual dimorphisms will be unaltered in long course of our evolutionary journey? Darwin's natural selection shapes the morphological forms of animals. Natural selection in the form of sexual selection has been one of the major factors in the expression of differential sexual features in the two sexes of majority of animal species. Reduction in average height of females than their male counterpart in majority of human races is the result of this selection. Preferential mating in humans based on body size variation will maintain this gap and the difference even may lead to more significant differences. As a consequence of this, females in remote future may show significantly shorter height compared to the male community.

A politician in India, once raised a question saying that how can a monkey change into a human being as claimed by evolution biologists. He said that none of us has seen anywhere in the forest or at any spot changing a monkey into a man. He refuted Darwin's theory that 
advocates evolutionary events. Remarks made by the minister triggered a wave of discussion by news and television media to counter minister's statement. In fact, understanding the ways by which evolutionary events take place, to see a species changing into another one needs lot of scientific knowledge. Such things can now be explained authentically, i.e., based on scientific evidences. The fossil remains are the direct evidences of human species or races that persisted in remote past and were much different from the present day humans. We have been able to articulate scientific history of our own species and also other vertebrates whose fossil remains have been obtained in better conditions. The process of evolution is extremely slow. To observe obvious changes in a species from its remote ancestral species needs millions of years of time span. How a monkey gave rise to an ape and a form of ape gave rise to a human race, needs understanding of the phenomenon of geographical isolation, changes in the genome by mutation and chromosomal rearrangement, reproductive isolation etc., and all these take a long period to occur. It can be, at the end said with complete certainty that such issues would not be the contentious one, once people would be imbibed with scientific knowledge of evolutionary events. 